\title{
What's going on following acute COVID-19? Clinical characteristics of patients in an out-patient rehabilitation program
}

\author{
Sergiu Albu ${ }^{\mathrm{a}, \mathrm{b}, \mathrm{c}, *}$, Nicolás Rivas Zozaya ${ }^{\mathrm{a}, \mathrm{b}, \mathrm{c}}$, Narda Murillo ${ }^{\mathrm{a}, \mathrm{b}, \mathrm{c}}$, Alberto García-Molina ${ }^{\mathrm{a}, \mathrm{b}, \mathrm{c}}$, \\ Cristian Andrés Figueroa Chacón ${ }^{\mathrm{a}, \mathrm{b}, \mathrm{c}}$ and Hatice Kumru ${ }^{\mathrm{a}, \mathrm{b}, \mathrm{c}}$ \\ ${ }^{a}$ Fundació Institut Guttmann, Institut Universitari de Neurorehabilitació adscrit a la UAB, \\ Badalona - Barcelona, Spain \\ ${ }^{\mathrm{b}}$ Universitat Autonoma de Barcelona, Bellaterra (Cerdanyola del Vallès), Spain \\ ${ }^{\mathrm{c}}$ Fundació Institut d'Investigació en Ciències de la Salut Germans Trias i Pujol, Badalona, Spain
}

Received 28 January 2021

Accepted 16 March 2021

\begin{abstract}
.
BACKGROUND: Coronavirus disease 2019 (COVID-19) patients present long-lasting physical and neuropsychological impairment, which may require rehabilitation.

OBJECTIVES: The current cross-sectional study characterizes post COVID-19 sequelae and persistent symptoms in patients in an outpatient rehabilitation program.

METHODS: Thirty patients [16 post-ICU and 14 non-ICU; median age $=54(43.8-62)$ years; 19 men] presenting sequelae and/or persistent symptoms ( $>3$ months after acute COVID-19) were selected of 41 patients referred for neurorehabilitation. Patients underwent physical, neuropsychological and respiratory evaluation and assessment of impact of fatigue and quality of life.

RESULTS: The main reasons for referral to rehabilitation were: fatigue (86.6\%), dyspnea (66.7\%), subjective cognitive impairment (46.7\%) and neurological sequelae (33.3\%). Post-ICU patient presented sequelae of critical illness myopathy and polyneuropathy, stroke and encephalopathy and lower forced vital capacity compared to non-ICU patients. Cognitive impairment was found in $63.3 \%$ of patients, with a similar profile in both sub-groups. Increased physical fatigue, anxiety and depression and low quality of life were prevalent irrespective of acute COVID-19 severity.

CONCLUSIONS: The variability of post COVID-19 physical and neuropsychological impairment requires a complex screening process both in ICU and non-ICU patients. The high impact of persistent symptoms on daily life activities and quality of life, regardless of acute infection severity, indicate need for rehabilitation.
\end{abstract}

Keywords: COVID-19, sequelae, persistent symptoms, neurological impairment, cognitive impairment

\section{Introduction}

The coronavirus disease 2019 (COVID-19) is considered primarily a respiratory disease caused by the novel severe acute respiratory syndrome coronavirus 2 (SARS-CoV-2). The clinical spectrum of

*Address for correspondence: Sergiu Albu, MD, PhD., Hospital de Neurorehabilitació Institut Guttmann, Camí Can Ruti s/n. Barcelona, 08916 Barcelona, Spain. Tel.: +34 9349777 00; Fax: +34 9349777 15; E-mail: salbu@guttmann.com. acute COVID-19 infection is heterogeneous, with approximately $15 \%$ of cases being asymptomatic (Byambasuren et al., 2020), whereas about $73 \%$ of patients present symptoms of acute respiratory infection (fever, cough, dyspnea) (Argenziano et al., 2020; Suleyman et al., 2020), with higher risk of severe illness in older adults, males and individuals with baseline comorbidities (hypertension, obesity, diabetes, chronic pulmonary or kidney disease) (Argenziano et al., 2020; Richardson et al., 2020). A relevant proportion of patients develop extrapulmonary 
manifestations such as musculoskeletal (fatigue and myalgia), cardiovascular (arrhythmias and cardiac injury), gastrointestinal (nausea, vomiting, diarrhea and abdominal pain) and neurological and psychiatric (olfactory and gustatory dysfunctions, headache, cognitive impairment, anxiety and depression) among the most common symptoms (AlSamman, Caggiula, Ganguli, Misak, \& Pourmand, 2020; Lechien et al., 2020; Negrini et al., 2020; Zhou et al., 2020). Only a small proportion of COVID-19 positive patients require admission to ICU due to severe hypoxemia and medical complications (acute respiratory distress syndrome, shock, myocardial dysfunction and acute kidney injury) (Phua et al., 2020). However, a significant number of individuals who survived severe COVID-19 may have persistent lung damage and long-term impairment of pulmonary function (Curci et al., 2020; Zhao, Shang, Song, \& al., 2020) as well as sequelae associated with critical illness and neurologic complications (cerebrovascular events, altered mental status, peripheral nervous system and muscular alteration) (Varatharaj et al., 2020). Although most patients are expected to recover within weeks after initial symptoms, at 1-1.5 months after discharge, a high proportion of individuals $(87.4 \%)$ reported persistence of at least 1 symptom, particularly fatigue and dyspnea and worsened quality of life (Carfi, Bernabei, Landi, \& Gemelli Against, 2020) and 34.3\% referred persistent cognitive complaints (Almeria, Cejudo, Sotoca, Deus, \& Krupinski, 2020).

The variability of sequelae and persistent symptoms requires a comprehensive screening and evaluation process by a multidisciplinary expert team and a range of tailored rehabilitation intervention (Wade, 2020). Early detection of persistent symptoms and sequelae in post COVID-19 patients and their inclusion in rehabilitation programs are imperative to reduce disability and to improve patients' quality of life and community reintegration.

In the current study we characterize persistent symptoms, physical, neurological and respiratory sequelae and their impact on daily life activities and quality of life in post COVID-19 patients included in an outpatient rehabilitation program. In addition, we evaluate the relationship between demographic characteristics, preexisting comorbidities, acute COV ID-19 severity (ICU vs non-ICU) and the spectrum of persistent symptoms and sequelae in this cohort. We hypothesize that both ICU and non-ICU patients will present multiple physical, neurological and respiratory persistent symptoms. We also expect that ICU patient will present with more severe symptoms, lower functional independency and quality of life and will initiate rehabilitation earlier after discharge compared to non-ICU patients.

\section{Methods}

This is a single center cross-sectional observational study on patients with persistent symptoms and/or sequelae of COVID-19 referred by a primary care physician or a specialist for outpatient rehabilitation at Institute Guttmann (Badalona, Barcelona, Spain).

Inclusion criteria were: adults $>18$ years with persistent symptoms or sequelae of COVID-19 infection confirmed by either PCR or serology.

Exclusion criteria were: previous neurological, psychiatric or severe medical condition; persistent symptoms of confirmed COVID-19 without indications for rehabilitation interventions (e.g. anosmia, dysgeusia, pain symptoms), clinically diagnosed COVID-19 with non-specific symptoms and negative PCR or serology.

A total of 41 individuals (16 post-ICU and 25 non-ICU) were evaluated in the outpatient department by a multidisciplinary COVID-19 team which included physicians (physical rehabilitation and neurology specialists), neuropsychologists and physical therapists. All recruited patients signed a written informed consent for participation in this research study. The study protocol was approved by the local Research Ethics Committee of and was carried out in accordance with the Declaration of Helsinki.

\subsection{Medical evaluation}

Before inclusion in the rehabilitation program, a structured medical evaluation gathered: demographic data; preexisting comorbidities; current smoking habits; acute COVID-19 symptoms and current respiratory, neurological and cognitive-affective symptoms from a checklist; duration of hospitalization and duration of ICU stay; required oxygen supplementation and ventilation support; acute COVID-19 treatment and complications.

Participants were asked to answer questionnaires evaluating the impact of the experienced physical, cognitive and social fatigue on daily life activities (Modified Fatigue Impact Scale, MFIS) (Larson, 2013), their sleep habits and perceived sleep quality (The Pittsburgh Sleep Quality Index) (Buysse, Reynolds, Monk, Berman, \& Kupfer, 1989) and perceived quality of life (WHOQOL-BREF questionnaire) (WHO, 1996). 


\subsection{Physical and respiratory evaluation}

Respiratory function was evaluated using a portable spirometer (Conter® SP10). The following parameters were measured: forced vital capacity (FVC); forced expiratory volume in 1 second $\left(\mathrm{FEV}_{1}\right)$; $\mathrm{FEV}_{1} / \mathrm{FVC}$ ratio; and the peek expiratory flow (PEF). Data were expressed as \% of the expected value according to their age, gender and height. A FVC and $\mathrm{FEV}_{1}>80 \%, \mathrm{a} \mathrm{FEV}_{1} / \mathrm{FVC}$ ratio $>0.7$ and $\mathrm{PEF}>80 \%$ of the expected value were considered normal (Garcia-Rio et al., 2013).

Maximal handgrip strength (MHS) was measured on the dominant non-affected hand or on the less affected hand using a hand dynamometer (Biometrics). MHS was recorded as the maximum value obtained from three consecutive trials and results were compared to normative values according to individual's age and gender (Massy-Westropp, Gill, Taylor, Bohannon, \& Hill, 2011).

The Short Physical Performance Test (SPPT) assessed lower extremity physical performance status based on gait speed, chair stand and balance tests (Bergland \& Strand, 2019).

The Functional Independence Measure (FIM) scale was used to assess measures of independence for self-care, including sphincter control, transfers, locomotion, communication, and social cognition (Mackintosh, 2009).

\subsection{Neuropsychological evaluation}

All patients underwent a baseline neuropsychological evaluation regardless of the presence of subjective cognitive complains. A set of neuropsychological tests was selected to cover the most representative aspects of cognitive functions.

Orientation in person, place and time was measured using the Barcelona Test which is based on the Benton Temporal Orientation Test (Benton, Hamsher, Varney, \& Spreen, 1983; Peña-Casanova, 1991). Immediate attention was assessed by means of Digit Span forward subtest of the Wechsler Adult Intelligence Scale III (Wechsler, 1997). Verbal memory was assessed using the Rey Auditory Verbal Learning Test (RAVLT) that includes three domains of verbal learning, long-term verbal memory, and verbal recognition (Schmidt, 1996). Working memory was evaluated employing the Digit Span backward subtest of the Wechsler Adult Intelligence Scale III (Wechsler, 1997). Executive control was evaluated using the PMR task (a Spanish version of the FAS letter fluency task) (Benton et al., 1983; Fortuny, 1999). In addition, patients were screened for symptoms of anxiety and depression (Hospital Anxiety and Depression Scale, HADS) (Herrero et al., 2003).

\subsection{Data analysis}

Statistical analyses were conducted with a commercial software package (SPSS, version 13.0; SPSS Inc., Chicago, IL, USA). Descriptive statistics characterized basic demographic and clinical characteristics of the patients enrolled in the study. The Chi-square test for independence was used to assess associations between categorical variables. The Shapiro-Wilk's test was used to examine the normality of distribution. The Mann-Whitney U test was used to compare differences between subgroups on age, respiratory function variables, MHS and sleep quality parameters. Quade's test for non-parametric ANCOVAs was conducted on FIM and SPPT as dependent variables and age and gender as covariables. General linear models were conducted to study between group differences in anxiety, depression, impact of fatigue and quality of life measures as dependent variables and age and gender as covariables. A Bonferroni correction was used for multiple comparisons. Spearman's correlation analyses were run to study relationships between continuous variables. Variables are presented as a median value with interquartile range (25-75\%). A two-tailed test with an alpha level of 0.05 was used for all analyses.

\section{Results}

Thirty individuals (16 post-ICU and 14 non-ICU) met the inclusion criteria and were recruited for

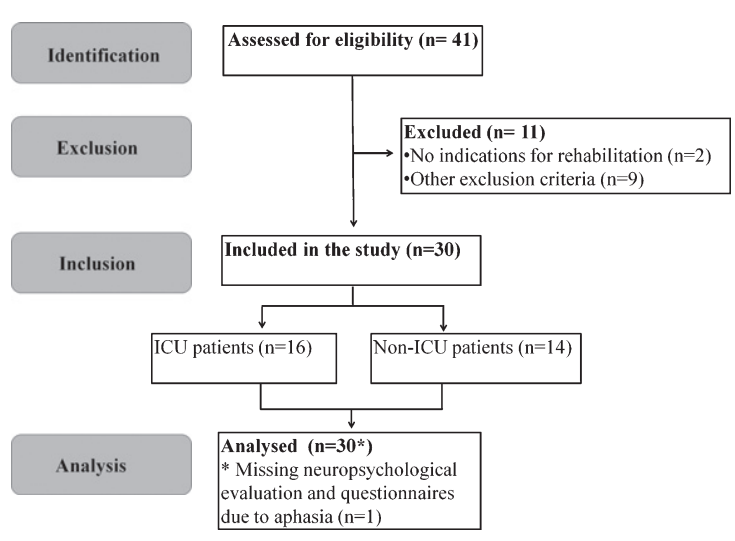

Fig. 1. STROBE flow diagram. 
the study. Three patients with clinically diagnosed COVID-19 with non-specific symptoms and negative PCR or serology and 1 patient with persistent symptoms of COVID-19 confirmed by positive PCR and previously known cognitive decline were included in a rehabilitation program but not enrolled in the current study. Other non-enrolled individuals were 4 patients who presented exacerbated symptoms of previously known affective disorders and had a negative PCR and serology for COVID-19; 2 patients with persistent symptoms (e.g. anosmia, dysguesia, pain symptoms) of COVID-19 confirmed by positive PCR and/or serology with no indications for rehabilitation; and 1 patient with symptoms of substance use disorders with negative PCR and serology for COVID-19 (Fig. 1).

\subsection{Baseline demographic and clinical characteristics}

The cohort of patients selected for the study consisted of: (I) the ICU subgroup which included 16 patients who developed severe COVID-19 and required admission to ICU and (II) the non-ICU subgroup included 14 patients of which 7 patients required admission to a conventional hospital unit (non-ICU) and 7 patients who were home confined (Table 1).

Compared to the non-ICU group, patients in the ICU subgroups were older $(p=0.001)$, predominantly male $(p=0.03)$ with preexisting obesity $(p=0.003)$ and hypertension $(p=0.01)$.

In the acute phase, bilateral pneumonia was the main reason for hospitalization in the ICU subgroup (16 patients) compared to bilateral or unilateral pneumonia (6 patients) and transitory loss of consciousness (1 patient) in the non-ICU subgroup. Acute COVID-19 medical and neurological complications were identified only in the ICU subgroup, which required a longer hospital stay and more aggressive oxygen supplementation and pharmacological treatment.

\subsection{Reason for referral to rehabilitation}

The main reasons for rehabilitation referred by patients during the baseline clinical evaluation, were: fatigue (86.6\%), dyspnea (66.7\%), cognitive impairment $(46.7 \%)$ and neurological sequelae $(33.3 \%)$ (Fig. 2).

On average, patients in the non-ICU subgroup started the rehabilitation 110.5 (89-124) days from the beginning of acute COVID-19 symptoms and 79 (64-90) days after discharge from conventional hospital unit, whereas in the ICU subgroup patients started the rehabilitation at 98 (95-112) days from the beginning of symptoms and 42 (8-64) days after hospital discharge. The time of rehabilitation start after beginning of symptoms and after hospital discharge were not statistically significant between sub-groups ( $p=0.26$ and $p=0.45$ respectively)

\subsection{Respiratory function}

Prevalence of alterations in spirometry varied among patients in the ICU vs non-ICU subgroup: low FVC $(<80 \%)$ was found in $31.25 \%$ vs $0 \%$; low $\mathrm{FEV}_{1}(<80 \%)$ in $35.71 \%$ vs $37.5 \%$; low $\mathrm{FEV}_{1} / \mathrm{FVC}$ ratio $(<0.7)$ in $7.1 \%$ vs $12.5 \%$; and low $\mathrm{PEF}(<80 \%)$ in $81.25 \%$ vs $50 \%$ of patients in each subgroup. The FVC was significantly lower in the ICU subgroup compared to the non-ICU subgroup ( $p=0.02$ ), but there were no statistically significant differences between subgroups in the $\operatorname{FEV}_{1}(p=0.26)$, $\mathrm{FEV}_{1} / \mathrm{FVC}(p=0.20)$ or PEF $(p=0.80)$ (Table 2, Fig. 3).

\subsection{Physical functioning and disability}

We did not find significant differences between subgroups for SPPT score $[\mathrm{F}(1,23)=0.88 ; p=0.36]$, FIM total score $[\mathrm{F}(1,25)=1.41 ; p=0.25]$, FIM motor sub-score $[\mathrm{F}(1,25)=1.99 ; p=0.17]$, FIM cognitive sub-score $[\mathrm{F}(1,25)=0.57 ; p=0.46]$ or MHS $[\mathrm{F}(1,25)=0.99 ; p=0.33]$ (Table 2). Furthermore, MHS correlated with SPPT $(\mathrm{R} 2=0.52 ; p=0.01)$ and FIM score $(\mathrm{R} 2=0.43 ; p=0.025)$ and PEF $(\mathrm{R} 2=0.45$, $p=0.02)$.

\subsection{Neuropsychological characteristics}

Results of the neuropsychological evaluation and answers to questionnaires were missing in one patient in the UCI subgroup due to communication limitations (non-fluent aphasia).

Overall, 12 of 14 patients reporting subjective cognitive complains (e.g. difficulties with focusing attention, concentration and short memory problems) and 13 of 15 patients without cognitive complains scored lower than expected by age and education in at least one cognitive test. However, reporting subjective cognitive complains was unrelated to altered cognitive tests $[\mathrm{X} 2(1)=0.17, p=0.68]$. Furthermore, 
Table 1

Health-related characteristics, clinical course and outcomes of acute infection in post COVID-19 patients

\begin{tabular}{|c|c|c|c|c|}
\hline Characteristics & Total & ICU sub-group & Non-ICU subgroup & $P$ value \\
\hline Number of patients & 30 & 16 & 14 & \\
\hline Gender (M/F) & $19 / 11$ & $13 / 3$ & $6 / 8$ & 0.03 \\
\hline Age (years) & $54(43.8-262)$ & $61.5(54-64.75)$ & $43.5(37.5-53.25)$ & 0.001 \\
\hline \multicolumn{5}{|l|}{ Coexisting conditions } \\
\hline Active smoking & 0 & 0 & 0 & NP \\
\hline Obesity & 13 & 9 & 4 & 0.13 \\
\hline BMI before COVID-19 & $27.52(23.74-31.47)$ & $30.28(26.9-33.50)$ & $23.80(22.10-30.32)$ & 0.003 \\
\hline BMI after COVID-19 & $24.30(21.88-28.05)$ & $25.57(23.04-29.39)$ & $23.20(21.09-28.05)$ & 0.15 \\
\hline Arterial Hypertension & 9 & 8 & 1 & 0.01 \\
\hline COPD & 3 & 2 & 1 & 0.63 \\
\hline Asthma & 3 & 1 & 2 & 0.47 \\
\hline Coronary heart disease & 3 & 2 & 1 & 0.63 \\
\hline Cerebrovascular disease & 2 & 1 & 1 & 0.92 \\
\hline Diabetes Mellitus type 1 & 0 & 0 & 0 & NP \\
\hline Diabetes Mellitus type 2 & 2 & 2 & 0 & 0.17 \\
\hline Immune disorders & 2 & 1 & 1 & 0.92 \\
\hline Kidney disease & 0 & 0 & 0 & NP \\
\hline Active Cancer & 0 & 0 & 0 & NP \\
\hline \multicolumn{5}{|l|}{ Acute COVID-19 course } \\
\hline Required hospital admission & 23 & 16 & 7 & 0.01 \\
\hline Required admission to ICU & 16 & 16 & 0 & 0.001 \\
\hline Length of hospital stay (days) & $37(15-69)$ & $57(36-86)$ & $11(4-15)$ & 0.001 \\
\hline Length of ICU stay (days) & $26(12-44)$ & $26(12-44)$ & 0 & 0.001 \\
\hline $\begin{array}{l}\text { Time from beginning of symptoms } \\
\text { to rehabilitation (days) }\end{array}$ & $103(93-116)$ & $98(95-112)$ & $110.5(89-124)$ & 0.26 \\
\hline $\begin{array}{l}\text { Time from hospital discharge } \\
\text { to rehabilitation (days) }\end{array}$ & $50(12-74)$ & $42(8-64)$ & $79(64-90)$ & 0.45 \\
\hline \multicolumn{5}{|l|}{$\begin{array}{l}\text { Clinical presentation and } \\
\text { medical complications }\end{array}$} \\
\hline Pneumonia & 24 & 16 & 8 & 0.002 \\
\hline ARDS & 6 & 6 & 0 & 0.01 \\
\hline Pulmonary emoblism & 4 & 4 & 0 & 0.04 \\
\hline Cardiac injury or arrhythmia & 3 & 3 & 0 & 0.09 \\
\hline Septic shock & 5 & 5 & 0 & 0.02 \\
\hline Acute kidney injury & 8 & 8 & 0 & 0.002 \\
\hline Weight loss $>10 \mathrm{~kg}$ & 11 & 11 & 0 & 0.001 \\
\hline \multicolumn{5}{|l|}{ Neurological complications } \\
\hline CIP/CIM only & 3 & 3 & 0 & 0.04 \\
\hline CIP/CIM +Brain stroke & 3 & 3 & 0 & 0.04 \\
\hline $\begin{array}{l}\text { CIP/CIM + Spinal } \\
\text { epidural hematoma }\end{array}$ & 1 & 1 & 0 & 0.28 \\
\hline Brain stroke only & 1 & 1 & 0 & 0.28 \\
\hline Encephalopathy & 2 & 2 & 0 & 0.17 \\
\hline \multicolumn{5}{|l|}{$\begin{array}{l}\text { O2 Supplementation and } \\
\text { Pharmacological treatments }\end{array}$} \\
\hline O2 support/NIV/IMV & $1 / 2 / 14$ & $0 / 1 / 14$ & $1 / 1 / 0$ & $0.28 / 0.92 / 0.001$ \\
\hline Antiretroviral & 12 & 12 & 0 & 0.001 \\
\hline Hydroxychloroquine & 17 & 12 & 5 & 0.03 \\
\hline Azithromycin & 15 & 10 & 5 & 0.14 \\
\hline Ceftriaxone & 12 & 10 & 2 & 0.007 \\
\hline Anti-IL-6 drug (Tocilizumab) & 7 & 7 & 0 & 0.005 \\
\hline Systemic glucocorticoids & 11 & 8 & 3 & 0.28 \\
\hline
\end{tabular}

Legend: ARDS: Acute Respiratory Distress Syndrome; COPD: Chronic obstructive pulmonary disease; CIP/CIM: Critical illness polyneuropathy/ Critical illness polyneuropathy myopathy; NIV: non-invasive ventilation; IMV: invasive mechanical ventilation. Variables are presented as absolute values and median value with interquartile range (25-75\%). The Chi-square test was used for categorical variables and the Mann-Whitney U test for continuous variables, to compare ICU vs non-ICU subgroups.

there was no significant association between previous admission to ICU and low scores on orientation $[\mathrm{X} 2(1)=0.97, p=0.33]$, attention $[\mathrm{X} 2(1)=1.01$, $p=0.32]$, verbal learning $[\mathrm{X} 2(1)=1.77, p=0.18]$, long-term verbal memory $[\mathrm{X} 2(1)=0.28, p=0.60]$, verbal recognition $[\mathrm{X} 2(1)=1.44, p=0.23]$, working 


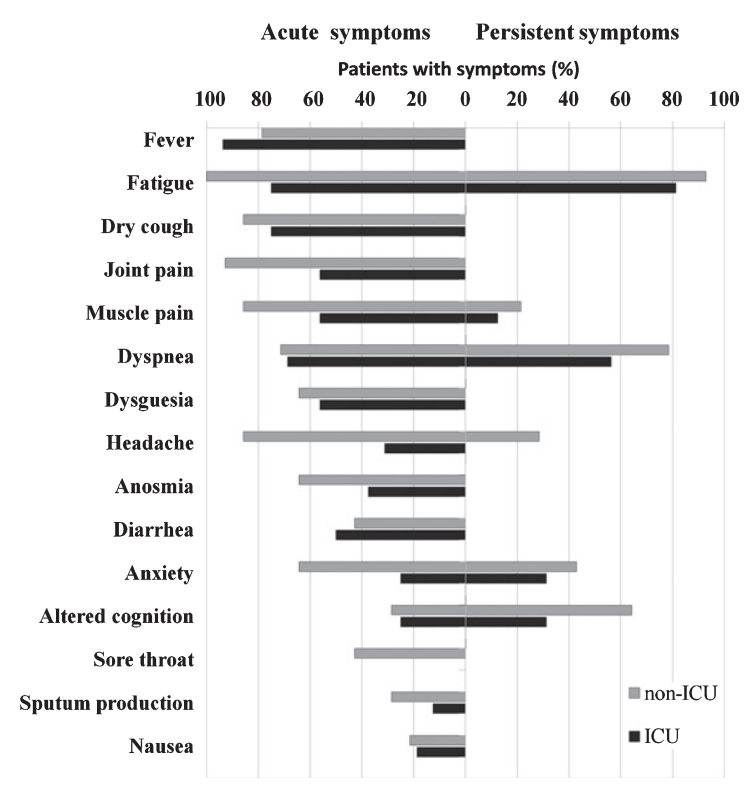

Fig. 2. Acute phase and persistent COVID-19 symptoms.

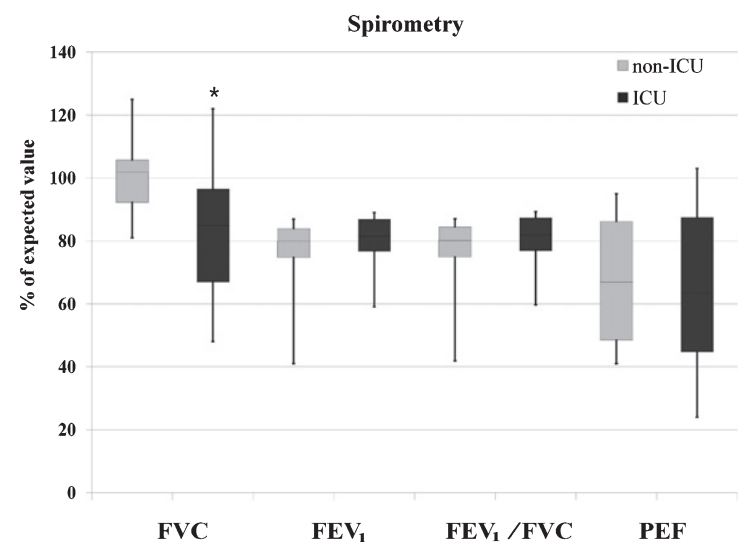

Fig. 3. Respiratory function. Legend: The upper, mid, and lower box edges represent the 75 th, 50 th, and 25 th percentiles, respectively. The whiskers represent maximum and minimum observed values. ${ }^{*} p<0.05$, Mann Whitney U test for ICU vs non-ICU subgroups comparison.

memory $[\mathrm{X} 2(1)=1.45, p=0.50]$ or executive control $[\mathrm{X} 2(1)=0.18, p=0.89]$ (Table 2, Fig. 4).

At cohort level, 11 patients had high anxiety and depression sub-scores ( $>8$ p) and 4 patients had increased depression score only. Furthermore, we found no significant differences in anxiety $[\mathrm{F}(1,25)=$ $2.91, p=0.10]$ or depression score $[\mathrm{F}(1,25)=3.77$, $p=0.06$ ] between subgroups (Table 2 ).

At cohort level, reporting subjective emotional distress was not associated with altered anxiety $[\mathrm{X} 2(1)=$ $0.86, p=0.36]$ or depression scores $[\mathrm{X} 2(1)=1.68$,

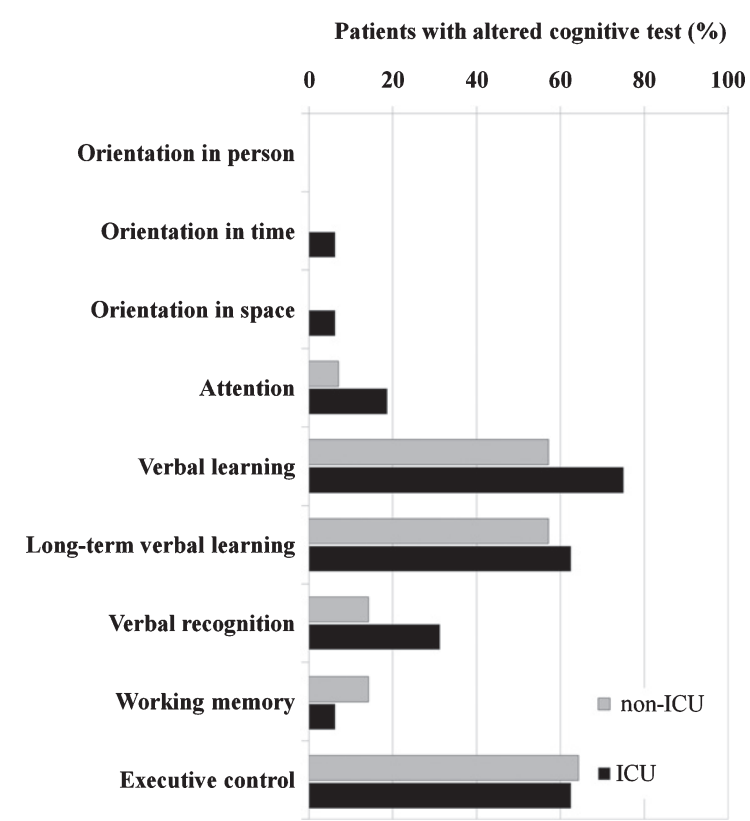

Fig. 4. Alterations in specific cognitive domains.

$p=0.19$ ]. However, patients presenting depression were more likely to have altered attention $[X 2(1)=$ 4.97, $p=0.026]$ and verbal recognition functions $[\mathrm{X} 2(1)=5.18, p=0.023]$.

\subsection{Sleep quality, impact of fatigue and quality of life}

Sleep quality was reported as being "fairly bad" or "bad" by 10 individuals in the non-ICU subgroup, and 2 patients were taking daily sleep medication. In the ICU subgroup, 2 patients reported "fairly bad" or "bad" sleep quality and 8 patients were taking sleep medication. Patients in the non-ICU subgroup had lower sleep quality $(p=0.01)$ and higher daytime dysfunction ( $p=0.046$ ) compared to the ICU subgroups but there were no significant differences in PSQI sub-scores for sleep latency, duration, efficacy, disturbance or medication use between subgroups ( $p>0.05$ for all comparisons) (Table 2).

At cohort level, most patients reported high impact of fatigue on physical and cognitive activities (Fig. 5), but we found no significant differences between subgroups on measures of impact of fatigue on physical $[\mathrm{F}(1,26)=1.22, p=0.28]$, cognitive $[\mathrm{F}(1,26)=1.22$, $p=0.28]$ or psychosocial activities $[\mathrm{F}(1,26)=0.75$, $p=0.40]$ (Table 2).

Overall, patients reported low quality of physical: 38 (19-50)\%; psychological: 50 (31-66) \%; social: 56 (44-75)\%; and environmental health: 56 
Table 2

Results of respiratory, physical and neuropsychological evaluation

\begin{tabular}{|c|c|c|c|c|}
\hline Characteristics & Total & ICU sub-group & Non-ICU sub-group & $P$ value \\
\hline \multicolumn{5}{|l|}{ Respiratory function } \\
\hline FVC & $91.5(81-104.5)$ & $85(67-96.5)$ & $102(92.25-105.75)$ & 0.02 \\
\hline FEV1 & $81.0(76.25-85.75)$ & $81.5(76.75-87)$ & $80(74.75-84)$ & 0.26 \\
\hline FEV1/FCV & $81.4(76.4-85.75)$ & $82.0(76.9-87.4)$ & $80.2(74.9-84.6)$ & 0.20 \\
\hline PEF & $65.5(47.75-86.25)$ & $63.5(44.75-87.5)$ & $67(48.5-86.25)$ & 0.80 \\
\hline \multicolumn{5}{|l|}{ Physical functioning } \\
\hline FIM total & $123(114-125)$ & $121.5(104-124.75)$ & $124(122-125)$ & 0.25 \\
\hline FIM motor & $89(81-91)$ & 88.5 (76.76-89.75) & $90(89-91)$ & 0.17 \\
\hline FIM cognitive & $34(33-35)$ & $34(33-35)$ & $34(32-35)$ & 0.46 \\
\hline MHS & $24(18-30)$ & $22.5(18-29.5)$ & $24(17-35)$ & 0.33 \\
\hline SPPT & $10.5(7.75-12)$ & $10(5-11)$ & $12(8-12)$ & 0.36 \\
\hline \multicolumn{5}{|l|}{ Neuropsychological } \\
\hline Orientation & $35(35-35)$ & $35(35-35)$ & $35(35-35)$ & 0.33 \\
\hline Attention & $6(5-6)$ & $5.5(5-6)$ & $6(5-7)$ & 0.32 \\
\hline Verbal learning & $44(36.5-50.75)$ & $40(33.75-44.75)$ & $47.5(40.75-54.5)$ & 0.18 \\
\hline Long-term verbal memory & $8.5(6-9.75)$ & $6(5.75-9)$ & $9(7.75-12)$ & 0.60 \\
\hline Verbal recognition & $12.5(11-14.75)$ & $12(10-14.25)$ & $13.5(11-15)$ & 0.23 \\
\hline Working memory & $4(4-5)$ & $4(4-5)$ & $4(4-5)$ & 0.50 \\
\hline Executive control & $40.5(27.5-47)$ & $29.5(21.75-47)$ & $44.5(34.5-50)$ & 0.89 \\
\hline \multicolumn{5}{|l|}{ HADS } \\
\hline Anxiety & $6(4-10)$ & $5(3-7)$ & $8.5(5.75-11.25)$ & 0.10 \\
\hline Depression & $8(3-9)$ & $4(3-9)$ & $9(6.5-10)$ & 0.06 \\
\hline \multicolumn{5}{|l|}{ Sleep quality } \\
\hline Quality & $1(0.5-2)$ & $1(0-1)$ & $2(1-3)$ & 0.012 \\
\hline Latency & $1(0-2)$ & $1(0-2)$ & $1(0-3)$ & 0.31 \\
\hline Duration & $1(0-1)$ & $1(0-1)$ & $1(0-2.25)$ & 0.45 \\
\hline Efficacy & $1(0-2)$ & $1(0-2)$ & $1(0-2)$ & 0.68 \\
\hline Disturbance & $1(1-2)$ & $1(1-1)$ & $1.5(1-2)$ & 0.06 \\
\hline Medication Use & $0(0-3)$ & $3(0-3)$ & $0(0-1.25)$ & 0.25 \\
\hline Daytime dysfunction & $1(0.5-2)$ & $1(0-2)$ & $2(1-2.25)$ & 0.046 \\
\hline \multicolumn{5}{|l|}{ Impact of fatigue } \\
\hline Physical & 80.55 (66.7-94.4) & 80.55 (63.9-94.4) & $81.9(68.75-95.1)$ & 0.28 \\
\hline Cognitive & $72.5(60-85)$ & $72.5(57.5-85)$ & $73.75(61.9-85.6)$ & 0.28 \\
\hline Social & $30(38.5-69.5)$ & $20(10-35)$ & $35(25-40)$ & 0.40 \\
\hline \multicolumn{5}{|l|}{ Quality of life } \\
\hline Physical & $38(19-50)$ & $38(31-63)$ & $25(19-45.5)$ & 0.30 \\
\hline Psychological & $50(31-66)$ & $56(38-69)$ & $44(23.5-57.75)$ & 0.46 \\
\hline Social & $56(44-75)$ & $69(44-75)$ & $50(25-70.5)$ & 0.14 \\
\hline Environmental & $56(50-63)$ & $56(50-75)$ & $56(50-63)$ & 0.48 \\
\hline
\end{tabular}

Variables are presented as absolute values and median value with interquartile range (25-75\%). The Chi-square test was used for categorical variables and the Mann-Whitney U test and the Quade's test for continuous variables, to compare ICU vs non-ICU subgroups.

(50-63)\%. However, the reported quality of physical health $[\mathrm{F}(1,25)=1.12, p=0.30]$, psychological health $[\mathrm{F}(1,25)=0.56, p=0.46]$, social relationship $[\mathrm{F}(1,25)=2.31, p=0.14]$ or environmental $[\mathrm{F}$ $(1,25)=0.53, p=0.48]$ did not differ between the ICU and non-ICU subgroups (Table 2). Measures of impact of fatigue correlated with aspects of affective impairment, sleep quality and quality of live (Table 3).

\section{Discussion}

The current cross-sectional study describes sequelae and persistent symptoms at more than 3 months after acute COVID-19 and their impact on activities of daily living, sleep and quality of life in patients in an outpatient rehabilitation program. Our main findings reveal that multiple persistent symptoms of COVID-19, such as fatigue, dyspnea and cognitive impairment, were main reasons for inclusion in the outpatient rehabilitation program irrespective the acute COVID-19 severity. Patients in the ICU subgroup were older, mostly male, and with obesity and arterial hypertension. Similarly, they more often presented with restrictive ventilatory impairment, single or multiple neurological sequelae of severe acute COVID-19 and were also more likely to be on treatment for sleep alterations. However, in contradiction to our hypothesis post-ICU patients did not 

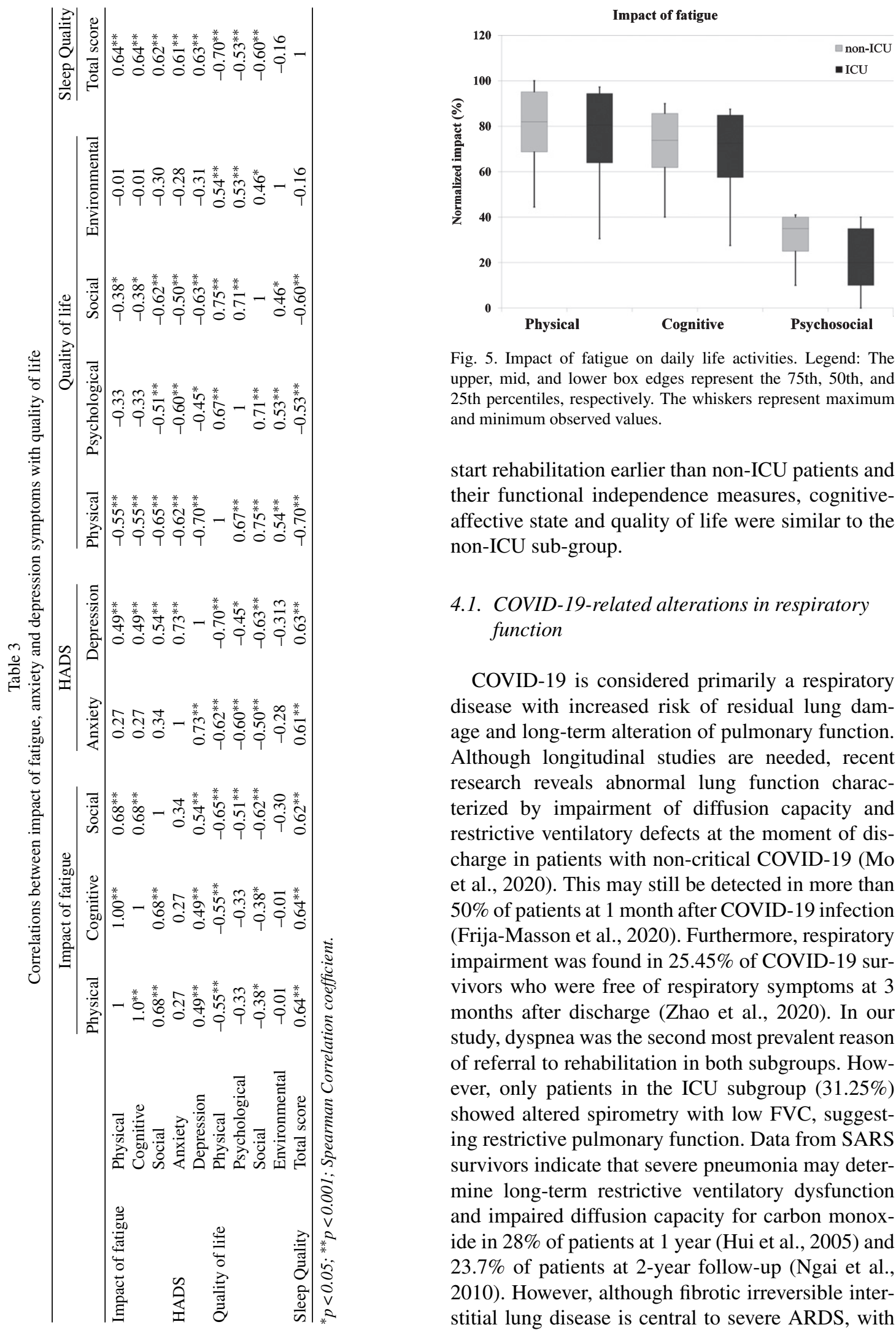

Fig. 5. Impact of fatigue on daily life activities. Legend: The upper, mid, and lower box edges represent the 75th, 50th, and 25 th percentiles, respectively. The whiskers represent maximum and minimum observed values.

start rehabilitation earlier than non-ICU patients and their functional independence measures, cognitiveaffective state and quality of life were similar to the non-ICU sub-group.

\subsection{COVID-19-related alterations in respiratory function}

COVID-19 is considered primarily a respiratory disease with increased risk of residual lung damage and long-term alteration of pulmonary function. Although longitudinal studies are needed, recent research reveals abnormal lung function characterized by impairment of diffusion capacity and restrictive ventilatory defects at the moment of discharge in patients with non-critical COVID-19 (Mo et al., 2020). This may still be detected in more than $50 \%$ of patients at 1 month after COVID-19 infection (Frija-Masson et al., 2020). Furthermore, respiratory impairment was found in 25.45\% of COVID-19 survivors who were free of respiratory symptoms at 3 months after discharge (Zhao et al., 2020). In our study, dyspnea was the second most prevalent reason of referral to rehabilitation in both subgroups. However, only patients in the ICU subgroup (31.25\%) showed altered spirometry with low FVC, suggesting restrictive pulmonary function. Data from SARS survivors indicate that severe pneumonia may determine long-term restrictive ventilatory dysfunction and impaired diffusion capacity for carbon monoxide in $28 \%$ of patients at 1 year (Hui et al., 2005) and $23.7 \%$ of patients at 2-year follow-up (Ngai et al., 2010). However, although fibrotic irreversible interstitial lung disease is central to severe ARDS, with 
higher risk in people of advanced age, more severe illness, longer ICU stay with need of mechanical ventilation (Gentile et al., 2020; Ojo, Balogun, Williams, \& Ojo, 2020), irreversible fibrotic interstitial lung disease in COVID-19 survivors remains speculative. Restrictive chest wall mobility in people with obesity and neuromuscular disorders such as critical illness myopathy and deconditioning may also contribute to ventilatory dysfunction (Melo, Silva, \& Calles, 2014; Nusair, 2020).

\subsection{COVID-19-related physical and neurological impairment}

Persistent fatigue was the most prevalent symptom of COVID-19 affecting $53.1 \%$ of patients discharged from the hospital after recovery from acute COVID19 (Carfi et al., 2020). Persistent fatigue is commonly reported by ARDS survivors at 1-year (Neufeld et al., 2020) and 4-year follow up (Lam et al., 2009). Main determinants of persistent fatigue may include ARDS severity, cytokine storm in the acute phase and the use of corticosteroids (Lam et al., 2009). Other studies suggest that ARDS severity and the length of ICU stay had little association with persistent fatigue, which seems to be conditioned by worse physical, cognitive, and mental health symptoms (Neufeld et al., 2020), in line with our findings. In our study, $81.25 \%$ patients in the ICU subgroup ad $92.86 \%$ in the non-ICU subgroup reported persistent fatigue at more than 3 months after acute COVID-19, and its prevalence and impact on daily life activities were independent of acute COVID-19 severity. Furthermore, the impact of physical fatigue correlated with higher depression score and low quality of physical and social life.

Severe COVID-19 patients referred for rehabilitation are characterized by older age, high prevalence of sequelae of multiple medical and neurological complications, preexisting comorbidities, long ICU stay and prolonged mechanical ventilation, consistent with other studies (Argenziano et al., 2020; Jurado et al., 2020; Petrilli et al., 2020; Richardson et al., 2020; Suleyman et al., 2020; Varatharaj et al., 2020; Wendel Garcia et al., 2020). In our study, $62.5 \%$ of patients who required admission to ICU presented single or multiple neurological complications. The diffuse and symmetrical muscle weakness due to CIP/CIM was the most prevalent neurological complication affecting $43.75 \%$ of patients in the ICU subgroup. Potential mechanisms of CIP/CIM include microcirculatory changes associated with systemic inflammation, increased vascular permeability, metabolic alterations, denervated muscles exposed to high-dose corticosteroids (Shepherd, Batra, \& Lerner, 2017), low-grade myotoxicity in patients with prolonged periods of mechanical ventilation and use of sedative drugs (Lonnqvist et al., 2020). Surprisingly, although CIP/CIM is the most common cause of neuromuscular weakness in the intensive care settings, affecting approximately $25 \%$ to $45 \%$ of patients admitted to the ICU (Lacomis, 2011), there are a few case reports of CIP/CIM in patients with COVID-19 (Bagnato et al., 2020; Tankisi et al., 2020). Furthermore, $25 \%$ of patients in the ICU subgroup presented sequelae of cerebral or spinal stroke in addition to CIP/CIM, probably due to preexisting vascular risk factors and complications of the critically ill patients (e.g., hypercoagulable state, shock, heart failure, arrhythmogenic cardiomyopathy, etc.), which increase the risk of stroke (Fifi \& Mocco, 2020; Montalvan, Toledo, \& Nugent, 2020). Although the presence of multiple neurological and medical complications determines longer hospital stays and increases the disability rates in the subacute COVID-19 phase, we found no differences in functional independence measures and lower extremity functioning between the ICU and non-ICU subgroups at $>3$ months after acute COVID-19.

\subsection{Neuropsychological functioning, sleep quality and quality of life}

During the acute COVID-19, patients may present alteration in personality, behavior, cognition and consciousness resulting from cerebrovascular events, encephalopathy and other complications such as sepsis and hypoxia and the use of sedative drugs (Helms et al., 2020; Rogers et al., 2020; Troyer, Kohn, \& Hong, 2020; Varatharaj et al., 2020). Zhou et al. (Zhou et al., 2020) report cognitive dysfunction in sustained attention in recovered COVID-19 patients as compared to healthy controls, which correlated with the level of inflammatory markers. Another study reported specific decline in attention, memory, language and praxis abilities in severe COVID-19 patients entering in the post-acute phase, which seemed to be associated with the length of ICU stay (Negrini et al., 2020). During the first months after hospital discharge $34.3 \%$ of patients referred persistent cognitive complaints and neuropsychological evaluation revealed alterations in memory domains, attention and semantic fluency, working memory and 
mental and phonetic fluency as well as high anxiety and depression scores (Almeria et al., 2020). In our study, patients in the ICU and non-ICU subgroup reported persistent subjective cognitive and affective symptoms (difficulties focusing attention, altered concentration, short term memory impairment and anxiety) beyond the considered recovery, constituting the third most prevalent reason for rehabilitation referral. However, their perception was not related with altered cognitive performance or clinically relevant anxiety or depression symptoms in clinical evaluation. Furthermore, whereas patients with more severe COVID-19 and longer ICU stay would be expected to have more severe cognitive impairment (Rogers et al., 2020) we did not observe any significant differences in the cognitive domains and affective measures between subgroups.

The COVID-19 pandemic can impact the mental health of people in the general population with higher rates of depression, anxiety and sleep disorders (Stanton et al., 2020). In our study, patients in the non-ICU subgroup more frequently reported untreated sleep alterations with lower sleep quality and higher daytime dysfunction compared to ICU patients, whereas higher anxiety and depression score correlated significantly with low physical, psychological and social quality of life at cohort level. Furthermore, at the cohort level, patients presenting with depression were more likely to have altered attention and verbal recognition functions. Subjective cognitive impairment is commonly seen in patients with sleep alterations (Bubbico et al., 2019) and affective symptoms (Hill et al., 2016; Zlatar et al., 2018), supporting the relevance of screening for sleep alterations and affective symptoms in post COVID-19 patients with subjective cognitive impairment.

\subsection{Implication for rehabilitation}

Our study showed that post COVID-19 patients may present multiple physical, neuropsychological and respiratory sequelae and persistent symptoms months after acute infection. The prevalence and severity of fatigue, cognitive-affective impairment and low quality of life, irrespective of the acute infection severity (ICU vs non-ICU patients) advocate complex evaluation and design of multidisciplinary rehabilitation. The European Respiratory Society and American Thoracic Society-coordinated International Task Force recommend the screening for treatable traits and more comprehensive assessment of rehabilitation needs, including physical as well as mental aspects, at 6-8 weeks after discharge (Spruit et al., 2020). The high prevalence of dyspnea, physical and cognitive fatigue could be important limiting factors for rehabilitation therefore should be considered when designing rehabilitation interventions to improve patients' tolerance and adherence to treatment.

\subsection{Limitations}

Our study has several limitations. We describe the profile of a specific subgroup of patients with post COVID-19 sequelae and persistent symptoms in individuals included in an outpatient rehabilitation program $>3$ months after acute phase therefore their clinical characteristic cannot be generalized to the broad population of COVID-19 patients. The study was conducted in a small population of post ICU and non-ICU COVID-19 patients therefore there is a possibility that comparisons between sub-groups were underpowered. We did not include patients with clinically diagnosed COVID-19 with criteria for physical and cognitive rehabilitation as we could not rule out other infective/non-infective etiology of symptoms. The study has a cross-sectional design therefore we do not have baseline (before COVID-19 infection) clinical measures and we cannot discard potential for preexisting respiratory, physical and cognitive-affective alterations. In addition, despite the high prevalence of dyspnea among post COVID19 patients, we did not evaluate its severity and impact on daily life activities.

\section{Conclusions}

Following acute COVID-19 infection, patients may present multiple physical, cognitive-affective and respiratory impairments. The high impact of persistent symptoms on daily life activities and quality of life advocate for a multidisciplinary evaluation and clinical profiling of post COVID-19 patients before rehabilitation interventions, regardless of the severity of acute infection.

\section{Acknowledgments}

We would like to thank our colleagues from Institute Guttmann: María Sainz Pelayo, Rocío Sanchez Carrión-Abascal, Antonia Enseñat Cantallops, Cristina Silvestre, Ivan Castro, Elisabeth Blanco, María Mateo and Mónica Salvador, for their clinical work in the post COVID-19 rehabilitation program. 


\section{Conflict of interest}

The authors declare no conflicts of interest.

\section{References}

Almeria, M., Cejudo, J. C., Sotoca, J., Deus, J., Krupinski, J. (2020). Cognitive profile following COVID-19 infection: Clinical predictors leading to neuropsychological impairment. Brain Behav Immun Health, 9, 100-163.

AlSamman, M., Caggiula, A., Ganguli, S., Misak, M., Pourmand, A. (2020). Non-respiratory presentations of COVID-19, a clinical review. Am J Emerg Med, 38(11), 2444-2454.

Argenziano, M. G., Bruce, S. L., Slater, C. L., Tiao, J. R., Baldwin, M. R., Barr, R. G.,... Chen, R. (2020). Characterization and clinical course of 1000 patients with coronavirus disease 2019 in New York: retrospective case series. BMJ, 369, m1996.

Bagnato, S., Boccagni, C., Marino, G., Prestandrea, C., D’Agostino, T., Rubino, F. (2020). Critical illness myopathy after COVID-19. Int J Infect Dis, 99, 276-278.

Benton, A. L., Hamsher, K., Varney, N. R., Spreen, O. S. (1983). Contributions to neuropsychological assessment. New York: Oxford University Press.

Bergland, A., Strand, B. H. (2019). Norwegian reference values for the Short Physical Performance Battery (SPPB): the Tromso Study. BMC Geriatr, 19(1), 216.

Bubbico, G., Di Iorio, A., Lauriola, M., Sepede, G., Salice, S., Spina, E.,... Tartaro, A. (2019). Subjective Cognitive Decline and Nighttime Sleep Alterations, a Longitudinal Analysis. Front Aging Neurosci, 11, 142.

Buysse, D. J., Reynolds, C. F., 3rd, Monk, T. H., Berman, S. R., Kupfer, D. J. (1989). The Pittsburgh Sleep Quality Index: a new instrument for psychiatric practice and research. Psychiatry Res, 28(2), 193-213.

Byambasuren, O., Cardona, M., Bell, K., Clark, J., McLaws, M.-L., Glasziou, P. (2020). Estimating the extent of asymptomatic COVID-19 and its potential for community transmission: systematic review and meta-analysis. medRxiv, 2020.2005.2010.20097543.

Carfi, A., Bernabei, R., Landi, F., Gemelli Against, C.-P.-A. C. S. G. (2020). Persistent Symptoms in Patients After Acute COVID19. JAMA.

Curci, C., Pisano, F., Bonacci, E., Camozzi, D. M., Ceravolo, C., Bergonzi, R.,... De Sire, A. (2020). Early rehabilitation in post-acute COVID-19 patients: data from an Italian COVID19 rehabilitation unit and proposal of a treatment protocol. A cross-sectional study. Eur J Phys Rehabil Med.

Fifi, J. T., Mocco, J. (2020). COVID-19 related stroke in young individuals. Lancet Neurol, 19(9), 713-715.

Fortuny, L. (1999). Manual de normas y procedimientos para la batería neuropsicológica en español.: Hove: Psychology Press.

Frija-Masson, J., Debray, M. P., Gilbert, M., Lescure, F. X., Travert, F., Borie, R.,... Bancal, C. (2020). Functional characteristics of patients with SARS-CoV-2 pneumonia at 30 days postinfection. Eur Respir J, 56(2).

Garcia-Rio, F., Calle, M., Burgos, F., Casan, P., Del Campo, F., Galdiz, J. B.,... Thoracic, S. (2013). Spirometry. Spanish Society of Pulmonology and Thoracic Surgery (SEPAR). Arch Bronconeumol, 49(9), 388-401.
Gentile, F., Aimo, A., Forfori, F., Catapano, G., Clemente, A., Cademartiri, F.,... Giannoni, A. (2020). COVID-19 and risk of pulmonary fibrosis: the importance of planning ahead. Eur $J$ Prev Cardiol, 27(13), 1442-1446.

Helms, J., Kremer, S., Merdji, H., Clere-Jehl, R., Schenck, M., Kummerlen, C.,... Meziani, F. (2020). Neurologic Features in Severe SARS-CoV-2 Infection. N Engl J Med, 382(23), 22682270.

Herrero, M. J., Blanch, J., Peri, J. M., De Pablo, J., Pintor, L., Bulbena, A. (2003). A validation study of the hospital anxiety and depression scale (HADS) in a Spanish population. Gen Hosp Psychiatry, 25(4), 277-283.

Hill, N. L., Mogle, J., Wion, R., Munoz, E., DePasquale, N., Yevchak, A. M., Parisi, J. M. (2016). Subjective Cognitive Impairment and Affective Symptoms: A Systematic Review. Gerontologist, 56(6), e109-e127.

Hui, D. S., Wong, K. T., Ko, F. W., Tam, L. S., Chan, D. P., Woo, J., Sung, J. J. (2005). The 1-year impact of severe acute respiratory syndrome on pulmonary function, exercise capacity, and quality of life in a cohort of survivors. Chest, 128(4), 2247-2261.

Jurado, A., Martin, M. C., Abad-Molina, C., Orduna, A., Martinez, A., Ocana, E.,... Molina, J. (2020). COVID-19: age, Interleukin-6, C-reactive protein, and lymphocytes as key clues from a multicentre retrospective study. Immun Ageing, 17, 22.

Lacomis, D. (2011). Neuromuscular disorders in critically ill patients: review and update. J Clin Neuromuscul Dis, 12(4), 197-218.

Lam, M. H., Wing, Y. K., Yu, M. W., Leung, C. M., Ma, R. C., Kong, A. P.,... Lam, S. P. (2009). Mental morbidities and chronic fatigue in severe acute respiratory syndrome survivors: longterm follow-up. Arch Intern Med, 169(22), 2142-2147.

Larson, R. D. (2013). Psychometric properties of the modified fatigue impact scale. Int J MS Care, 15(1), 15-20.

Lechien, J. R., Chiesa-Estomba, C. M., De Siati, D. R., Horoi, M., Le Bon, S. D., Rodriguez, A.,... Saussez, S. (2020). Olfactory and gustatory dysfunctions as a clinical presentation of mild-to-moderate forms of the coronavirus disease (COVID19): a multicenter European study. Eur Arch Otorhinolaryngol, 277(8), 2251-2261.

Lonnqvist, P. A., Bell, M., Karlsson, T., Wiklund, L., Hoglund, A. S., Larsson, L. (2020). Does prolonged propofol sedation of mechanically ventilated COVID-19 patients contribute to critical illness myopathy? Br J Anaesth, 125(3), e334-e336.

Mackintosh, S. (2009). Functional independence measure. Aust $J$ Physiother, 55(1), 65.

Massy-Westropp, N. M., Gill, T. K., Taylor, A. W., Bohannon, R. W., Hill, C. L. (2011). Hand Grip Strength: age and gender stratified normative data in a population-based study. BMC Res Notes, 4, 127.

Melo, L. C., Silva, M. A., Calles, A. C. (2014). Obesity and lung function: a systematic review. Einstein (Sao Paulo), 12(1), 120125.

Mo, X., Jian, W., Su, Z., Chen, M., Peng, H., Peng, P.,... Li, S. (2020). Abnormal pulmonary function in COVID-19 patients at time of hospital discharge. Eur Respir J, 55(6).

Montalvan, V., Toledo, J., Nugent, K. (2020). Mechanisms of Stroke in Coronavirus Disease 2019. J Stroke, 22(2), 282-283.

Negrini, F., Ferrario, I., Mazziotti, D., Berchicci, M., Bonazzi, M., de Sire, A.,... Zapparoli, L. (2020). Neuropsychological features of severe hospitalized COVID-19 patients at clinical 
stability and clues for post-acute rehabilitation. Arch Phys Med Rehabil.

Neufeld, K. J., Leoutsakos, J. S., Yan, H., Lin, S., Zabinski, J. S., Dinglas, V. D.,... Needham, D. M. (2020). Fatigue Symptoms During the First Year Following ARDS. Chest, 158(3), 9991007.

Ngai, J. C., Ko, F. W., Ng, S. S., To, K. W., Tong, M., Hui, D. S. (2010). The long-term impact of severe acute respiratory syndrome on pulmonary function, exercise capacity and health status. Respirology, 15(3), 543-550.

Nusair, S. (2020). Abnormal carbon monoxide diffusion capacity in COVID-19 patients at time of hospital discharge. Eur Respir $J, 56(1)$.

Ojo, A. S., Balogun, S. A., Williams, O. T., Ojo, O. S. (2020). Pulmonary Fibrosis in COVID-19 Survivors: Predictive Factors and Risk Reduction Strategies. Pulm Med, 6175964.

Peña-Casanova, J. (1991). Programa integrado de exploración neuropsicológica 'Test Barcelona'. Normalidad, semiología y patología neuropsicológicas.. Barcelona: Masson.

Petrilli, C. M., Jones, S. A., Yang, J., Rajagopalan, H., O’Donnell, L., Chernyak, Y.,... Horwitz, L. I. (2020). Factors associated with hospital admission and critical illness among 5279 people with coronavirus disease 2019 in New York City: prospective cohort study. BMJ, 369, m1966.

Phua, J., Weng, L., Ling, L., Egi, M., Lim, C. M., Divatia, J. V.,... Asian Critical Care Clinical Trials, G. (2020). Intensive care management of coronavirus disease 2019 (COVID-19): challenges and recommendations. Lancet Respir Med, 8(5), 506-517.

Richardson, S., Hirsch, J. S., Narasimhan, M., Crawford, J. M., McGinn, T., Davidson, K. W.,... Zanos, T. P. (2020). Presenting Characteristics, Comorbidities, and Outcomes Among 5700 Patients Hospitalized With COVID-19 in the New York City Area. JAMA.

Rogers, J. P., Chesney, E., Oliver, D., Pollak, T. A., McGuire, P., Fusar-Poli, P.,... David, A. S. (2020). Psychiatric and neuropsychiatric presentations associated with severe coronavirus infections: a systematic review and meta-analysis with comparison to the COVID-19 pandemic. Lancet Psychiatry, 7(7), 611-627.

Schmidt, M. (1996). Rey Auditory Verbal Learning Test.. Los Angeles, CA: Western Psychological Services.

Shepherd, S., Batra, A., Lerner, D. P. (2017). Review of Critical Illness Myopathy and Neuropathy. Neurohospitalist, 7(1), 4148.

Spruit, M. A., Holland, A. E., Singh, S. J., Tonia, T., Wilson, K. C., Troosters, T. (2020). COVID-19: Interim Guidance on Rehabilitation in the Hospital and Post-Hospital Phase from a European Respiratory Society and American Thoracic Societycoordinated International Task Force. Eur Respir J.
Stanton, R., To, Q. G., Khalesi, S., Williams, S. L., Alley, S. J., Thwaite, T. L.,... Vandelanotte, C. (2020). Depression, Anxiety and Stress during COVID-19: Associations with Changes in Physical Activity, Sleep, Tobacco and Alcohol Use in Australian Adults. Int J Environ Res Public Health, 17(11).

Suleyman, G., Fadel, R. A., Malette, K. M., Hammond, C., Abdulla, H., Entz, A.,... Brar, I. (2020). Clinical Characteristics and Morbidity Associated With Coronavirus Disease 2019 in a Series of Patients in Metropolitan Detroit. JAMA Netw Open, 3(6), e2012270.

Tankisi, H., Tankisi, A., Harbo, T., Markvardsen, L. K., Andersen, H., Pedersen, T. H. (2020). Critical illness myopathy as a consequence of Covid-19 infection. Clin Neurophysiol, 131(8), 1931-1932.

Troyer, E. A., Kohn, J. N., Hong, S. (2020). Are we facing a crashing wave of neuropsychiatric sequelae of COVID-19? Neuropsychiatric symptoms and potential immunologic mechanisms. Brain Behav Immun, 87, 34-39.

Varatharaj, A., Thomas, N., Ellul, M. A., Davies, N. W. S., Pollak, T. A., Tenorio, E. L.,... Coro Nerve Study, G. (2020). Neurological and neuropsychiatric complications of COVID-19 in 153 patients: a UK-wide surveillance study. Lancet Psychiatry.

Wade, D. T. (2020). Rehabilitation after COVID-19: an evidencebased approach. Clin Med (Lond), 20(4), 359-365.

Wechsler, D. (1997). WAIS-III manual. San Antonio, TX: Psychological Corporation.

Wendel Garcia, D., Fumeaux, T., Guerci, P., Heuberger, D., Montomoli, J., Roche-Campo, F.,... Hilty, M. P. (2020). Prognostic factors associated with mortality risk and disease progression in 639 critically ill patients with COVID-19 in Europe: Initial report of the international RISC-19-ICU prospective observational cohort. E Clinical Medicine.

WHO. (1996). Administration and scoring. Field trial version. Retrieved from https://www.who.int/mental_health/ media/en/76.pdf?ua=1.

Zhao, Y., Shang, Y., Song, W., al., e. (2020). Follow-up study of the pulmonary function and related physiological characteristics of COVID-19 survivors three months after recovery. E Clinical Medicine.

Zhou, H., Lu, S., Chen, J., Wei, N., Wang, D., Lyu, H.,... Hu, S. (2020). The landscape of cognitive function in recovered COVID-19 patients. J Psychiatr Res, 129, 98-102.

Zlatar, Z. Z., Muniz, M. C., Espinoza, S. G., Gratianne, R., Gollan, T. H., Galasko, D., Salmon, D. P. (2018). Subjective Cognitive Decline, Objective Cognition, and Depression in Older Hispanics Screened for Memory Impairment. J Alzheimers Dis, 63(3), 949-956. 